\title{
Supplementation of natural minerals on the performance of broiler
}

\author{
Z Ahamed ${ }^{1}$, SC Das², B Dey', MR Azad ${ }^{1}$ and KMS Islam ${ }^{3}$
}

${ }^{1}$ C. P. Bangladesh Co., Ltd. E-236, Ward - 7, Chandra, Kaliakoir, Gazipur, Bangladesh; ${ }^{2}$ Department of Poultry Science, Bangladesh Agricultural University, Mymensingh 2202; ${ }^{3}$ Department of Animal Nutrition, Bangladesh Agricultural University, Mymensingh 2202

\begin{abstract}
An experiment was conducted to evaluate the efficacy of AZOMITE ${ }^{\circledR}$ as source of natural minerals on growth performance of commercial broiler. A total of 1,020 day-old Cobb 500 male broiler chicks were randomly allocated to 5 dietary treatments with 6 replications per treatment having 34 chicks in each pen. The five dietary treatments were: (i) basal diet without AZOMITE ${ }^{\circledR}$, (ii) basal diet with $0.25 \%$ AZOMITE $^{\circledR}$, (iii) basal diet with $0.50 \%$ AZOMITE ${ }^{\circledR}$ which was recommended by manufacturer, (iv) basal diet with $0.75 \%$ AZOMITE $^{\circledR}$ and $(v)$ basal diet with $1.0 \%$ AZOMITE $^{\circledR}$. Broilers were reared in open-sided gable type house for a period of 32 days. Results showed that the supplementation of AZOMITE $^{\circledR}$ had significant effect on growth performance $(p<0.01)$ of broilers. Live body weight $(L B W)$, body weight gain (BWG), average daily gain (ADG), feed intake (FI), feed conversion ratio (FCR), European efficiency factor (EEF) and gain cost of the birds fed various levels of AZOMITE ${ }^{\circledR}$ were significantly improved as compared to the control. Meat characteristics results indicated that the treatments had no significant effect on dressing percent, thigh, drumsticks and breast percentage among the dietary groups. Taken together, it may be concluded that the supplementation of AZOMITE ${ }^{\circledR}$ in commercial broiler at 0.50 to $1.0 \%$ improved live weight, feed intake and feed conversion ratio of commercial broilers.
\end{abstract}

Key words: azomite, broiler, natural minerals, volcanic ash

Bangladesh Animal Husbandry Association. All rights reserved.

Bang. J. Anim. Sci. 2019. 48 (2):92-98

\section{Introduction}

Quality of poultry feed is an important precondition to achieve optimal production results, and preservation of the health of birds, especially in intensive poultry production; hence it is necessary to control both raw materials and finished feed products (Karovic et al., 2013). Besides, the key nutrients in commercial poultry feed such as proteins, carbohydrates, fats, and certain vitamins, many other macro and micro minerals are also considered as indispensable part of the bird's nutritional requirements. Minerals are chemical constituents used by the biological systems in many ways. Although, the mineral constituents do not yield energy for the birds, they have pivotal roles in many biological activities in the body (Malhotra, 1998). The importance of mineral elements in human, animal and plant nutrition has been well recognized (Underwood, 1971). Khatun et al., (2018) reported that two forms of organic trace minerals such as propionate and proteinate improved performance of commercial broilers over those of in-organic trace minerals. Every form of living matter requires these inorganic elements or minerals for their normal life processes (Hays and Swenson, 1985; Ozcan, 2003). Unlike other nutrients, mineral elements cannot be synthesized by living organisms (McDowell, 2003), thus these elements must be supplied from the exogenous sources through diets. Minerals have four broad functions like structural, physiological, catalytic and hormonal or regulatory. At early of $21^{\text {st }}$ century, the only supplemental minerals that were generally recognized as of value and administered to the livestock very infrequently were common salt $(\mathrm{NaCl})$, iron $(\mathrm{Fe})$, or iodine $(\mathrm{I})$.

Nowadays, minerals make up roughly half of the 40 or so required nutrients in broiler feeds, and these minerals, based on their dietary requirement, may be classified as essential major or macro-minerals (calcium, phosphorus, potassium, sodium and magnesium) and trace or micro-minerals. Nielsen (1985) indicated that between 1970 and 1985, at least 11 elements were added to the list of elements essential in animal nutrition. Chromium has been legally approved in several countries around the world 


\section{Natural minerals on broiler performance}

(200-400 parts per billion additions to the diet may be representative) for some animal species and purposes (Qinghua, 1996). Uthus and Seaborn (1996) also suggested the aluminum, rubidium and germanium as essential for the bird's diet. Tungsten, in the form of tungstate, exhibits a significant anti-hyperglycemic effect in both type 1 and 2 diabetic animals (Liu et al., 2004). Nielsen (1996) suggested that the term ultra-trace elements could be applied to at least 20 elements that have established estimated or suspected requirements or have beneficial effects. AZOMITE ${ }^{\circledR}$ has the ability to promote growth of commercial broiler (Hooge, 2008). Until now, there have been no or little research works was carried out focusing the supplementation of AZOMITE $^{\circledR}$ in broiler chickens; therefore, the effects and optimal level for dietary supplementation of AZOMITE $^{\circledR}$ in broiler diets under local environment is yet unknown. Thus, the objective of this experiment was to know the effect of AZOMITE $^{\circledR}$ as partial supplementation of macro and micro minerals in commercial broiler.

\section{Materials and Methods}

\section{Experimental birds}

A total of 1020 male Cobb 500 commercial broiler DOCs were used in this experiment having average weight of $46.59 \mathrm{~g} / \mathrm{chick}$, divided into five groups, one control group and four treatment groups with 204 chicks per group. AZOMITE ${ }^{\circledR}$ was fed at five levels increasing from $0 \%$ to $1.0 \%$ i.e. increments of $0.25 \%$. The duration of experiment was 32 days. The chicks were collected from a reputed hatchery by grading, sexing and weighing individually to maintain homogeneity of experimental units. Chicks were received at the day of hatch and upon arrival in $2.13 \mathrm{~m} \times 2.13 \mathrm{~m}$ floor pens at 7.5 birds $/ \mathrm{m}$. Charcoal brooders were used in each pen for brooding. The broiler was exposed to a continuous lighting of 23 hours light and 1 hour dark. Rice husk at a depth of $5 \mathrm{~cm}$ were placed as litter material. Two round tube feeders and one automatic bell drinker were provided in each pen. The feeders and drinkers were fixed in such a way that the birds were able to eat and drink conveniently. Feeders were washed and cleaned everyday while drinkers were cleaned twice in a day; morning and afternoon. Feed and water were supplied on adlibitum basis. Birds were vaccinated against Newcastle Disease (ND), Infectious Bronchitis (IB) and Infectious Bursal Diseases (IBD) as a part of disease prevention program. Vaccines were used as per manufacturers' instructions.

\section{Experimental diets}

Diets were formulated by using commonly available feed ingredients. The broiler diets were formulated for two phases namely starter and grower (Table 1 and 2). Diets were formulated in mash form. The nutrient requirements (ME, $C P$, $\mathrm{CF}, \mathrm{EE}, \mathrm{Ca}, \mathrm{P}$, Lysine and Methionine) were satisfied as recommended for Cobb-500 broiler strain diet and also same for all treatment except AZOMITE $^{\circledR}$. Chemical analysis of experimental diets was done at the laboratory of C.P. Bangladesh Co., Ltd., Gazipur, Bangladesh.

\section{Data collection and record keeping}

All the data of birds' weight, feed intake and mortality were recorded weekly. Using this data, live body weight (LBW), body weight gain (BWG), average daily gain (ADG), feed intake (FI), feed conversion ratio (FCR), survivability (\%), European efficiency factor (EEF-factor standardizes technical results, taking into account FCR, mortality and daily gain ) and gain cost (feed cost in BDT/kg meat) were calculated for each period of growth. During entire experimental period, the temperature and humidity of experimental house were recorded three times in a day (8.00AM, 1.00PM, 5.00PM) with the help of an automatic hygro-thermometer (Smart Sensor, Model AR 867, Taiwan). At the end of trial, two broilers having body weight near to pen average weight were taken from each pen for recording meat yield parameters. The birds were killed and allowed to bleed for 2 minutes and immersed in hot water $\left(51-55^{\circ} \mathrm{C}\right)$ for 120 seconds in order to lose the feathers and this procedure was called semi-scalding. The feathers were removed manually. Then head, shank, viscera, giblet (heart, liver and gizzard) and abdominal fat were removed for determination of meat yield parameters. Dressed broilers were cut into different parts such as breast, thigh, drumstick, wing and back. Finally, records were kept on weight of dressed broilers, breast meat, thigh, drumstick, wing, liver, heart, gizzard, spleen, head and neck.

\section{Data analysis}

Collected data were subjected to analysis of variance (ANOVA) in a completely randomized design (CRD) using SAS version 9.0 (2002) statistical computer package programs. The difference between means of groups was separated by New Duncan's Multiple Range Test (Steel and Torrie, 1980). Statement of statistical significance were based on $\mathrm{p}<0.05$. 


\section{Results and Discussion}

\section{Growth performances of broilers}

Broiler receiving AZOMITE $^{\circledR}$ at $0.25 \%, 0.50 \%$, $0.75 \%$ or $1.00 \%$ weighed significantly higher $(\mathrm{P}<0.01)$ than the control (Table 3$)$. At the end of 32 days, the highest live weight $(1628 \mathrm{~g} / \mathrm{bird})$ was found in broilers fed with AZOMITE $^{\circledR}$ at $0.75 \%$ level. This was followed by broilers feeding AZOMITE ${ }^{\circledR}$ at $0.50 \%$ (1607.52g/bird), $1.00 \%$ (1582 g/bird), $0.25 \%$ (1545 g/bird) and $0.0 \%$ (1206 g/bird) respectively. However, there were no significant differences observed among the broilers of various diet groups at 3 weeks of age. McNaughton et al. (2011) reported that
$0.5 \% \quad$ AZOMITE $^{\circledR}$ supplementation had no significant effect on live body weight $(p>0.05)$ but significant effect on feed conversion ratio $(p<0.01)$ on broiler. Hooge (2008) compiled 26 comparisons of negative control diets versus AZOMITE $^{\circledR}$ supplemented diets and concluded that AZOMITE $^{\circledR}$ at $0.5-1.0 \%$ improved body weight, feed conversion ratio and survivability. The result of this study agreed well with the findings of McNaughton et al. (2011) and Hooge (2008). In the present study, we found that growth performance of broiler chickens were significantly improved feeding 0.5 to $1 \%$ AZOMITE $^{\circledR}$.

Table 1: Ingredient and nutrient composition of starter ration (0-21 day)

\begin{tabular}{|c|c|c|c|c|c|}
\hline \multirow{2}{*}{ Ingredients ( $\%)$} & \multicolumn{5}{|c|}{ Treatments ( \% AZOMITE ${ }^{\circledR}$ ) } \\
\hline & 0.00 & 0.25 & 0.50 & 0.75 & 1.00 \\
\hline Corn & 47.47 & 47.47 & 47.47 & 47.47 & 47.47 \\
\hline Soybean meal $45 \%$ & 34.60 & 34.60 & 34.60 & 34.60 & 34.60 \\
\hline Rice bran fresh $12 \%$ & 5.00 & 5.00 & 5.00 & 5.00 & 5.00 \\
\hline Protein Conc. $48 \%$ & 3.26 & 3.26 & 3.26 & 3.26 & 3.26 \\
\hline Mustard oil cake $36 \%$ & 3.00 & 3.00 & 3.00 & 3.00 & 3.00 \\
\hline Lecithinized fat & 2.50 & 2.50 & 2.50 & 2.50 & 2.50 \\
\hline Palm fatty acid & 2.00 & 2.00 & 2.00 & 2.00 & 2.00 \\
\hline Limestone $37 \%$ & 0.68 & 0.68 & 0.68 & 0.68 & 0.68 \\
\hline Monocalcium phosphate & 0.41 & 0.41 & 0.41 & 0.41 & 0.41 \\
\hline DL- Methionine 99\% & 0.39 & 0.39 & 0.39 & 0.39 & 0.39 \\
\hline Common Salt & 0.30 & 0.30 & 0.30 & 0.30 & 0.30 \\
\hline Vitamin premix & 0.10 & 0.10 & 0.10 & 0.10 & 0.10 \\
\hline Mineral premix & 0.10 & 0.10 & 0.10 & 0.10 & 0.10 \\
\hline L-Lysine $78 \%$ & 0.10 & 0.10 & 0.10 & 0.10 & 0.10 \\
\hline Coccidiostat & 0.06 & 0.06 & 0.06 & 0.06 & 0.06 \\
\hline Choline Chloride $60 \%$ & 0.03 & 0.03 & 0.03 & 0.03 & 0.03 \\
\hline AZOMITE $^{\circledR}$ & ---- & 0.25 & 0.50 & 0.75 & 1.00 \\
\hline \multicolumn{6}{|c|}{ Analysed values of the nutrients } \\
\hline Moisture (\%) & 10.34 & 10.64 & 10.49 & 10.48 & 10.44 \\
\hline Crude Protein (\%) & 21.70 & 21.80 & 21.83 & 21.83 & 21.86 \\
\hline Crude Fibre (\%) & 3.62 & 4.05 & 3.54 & 3.60 & 3.83 \\
\hline Crude Fat (\%) & 6.24 & 6.35 & 6.68 & 6.16 & 6.74 \\
\hline Ash (\%) & 7.32 & 7.21 & 7.00 & 7.37 & 7.71 \\
\hline Salt (\%) & 0.32 & 0.30 & 0.29 & 0.30 & 0.30 \\
\hline
\end{tabular}




\section{Natural minerals on broiler performance}

Feed intake, feed conversion and economics of AZOMITE ${ }^{\circledR}$ supplementation

Feed intake, feed conversion and economics of AZOMITE ${ }^{\circledR}$ supplementation is shown in Table 4. Cumulative feed intake per broiler from 0-32 days of age revealed that AZOMITE $^{\circledR}$ supplemented diets significantly increased feed intake $(p<0.01)$ compared to control group. The broilers in control diet had lowest feed intake while $0.25, \quad 0.50, \quad 0.75, \quad 1.00 \% \quad$ AZOMITE $^{\circledR}$ supplemented diets had higher feed intake at 2232 days $(p<0.01)$. However, there were no significant differences observed among the treatment groups with increasing levels of AZOMITE $^{\circledR}(0.25-1.00 \%)$. The broilers in control diet had poor FCR while $0.25,0.50,0.75$ and $1.00 \% \quad$ AZOMITE $^{\circledR}$ supplemented diets had improved at $22-32$ days $(p<0.01)$. Cumulative FCR of the experimental broilers from 0-32 days of age revealed that AZOMITE $^{\circledR}$ supplemented diets significantly improved feed conversion ratio $(p<0.01)$ compared to control group. There were no significant differences among the treatment groups with increasing level of AZOMITE $^{\circledR}$ (0.25$1.00 \%)$.

Table 2. Ingredient and nutrient composition of grower ration (22-32 day)

\begin{tabular}{|c|c|c|c|c|c|}
\hline \multirow{2}{*}{ Ingredients $(\%)$} & \multicolumn{5}{|c|}{ Treatment $\left(\%\right.$ AZOMITE $\left.^{\circledR}\right)$} \\
\hline & 0.00 & 0.25 & 0.50 & 0.75 & 1.00 \\
\hline Corn & 44.04 & 44.04 & 44.04 & 44.04 & 44.04 \\
\hline Protein Conc. $51 \%$ & 1.90 & 1.90 & 1.90 & 1.90 & 1.90 \\
\hline Soybean meal $49 \%$ & 24.20 & 24.20 & 24.20 & 24.20 & 24.20 \\
\hline Mustard oil cake $36 \%$ & 5.00 & 5.00 & 5.00 & 5.00 & 5.00 \\
\hline Rice bran fresh $11.5 \%$ & 5.00 & 5.00 & 5.00 & 5.00 & 5.00 \\
\hline Rice bran solvent $18 \%$ & 9.97 & 9.97 & 9.97 & 9.97 & 9.97 \\
\hline Palm fatty acids & 2.00 & 2.00 & 2.00 & 2.00 & 2.00 \\
\hline Lecithinized fat & 4.80 & 4.80 & 4.80 & 4.80 & 4.80 \\
\hline Monocalcium phosphate & 0.91 & 0.91 & 0.91 & 0.91 & 0.91 \\
\hline Limestone $37 \%$ & 1.10 & 1.10 & 1.10 & 1.10 & 1.10 \\
\hline Common Salt & 0.33 & 0.33 & 0.33 & 0.33 & 0.33 \\
\hline DL- Methionine $99 \%$ & 0.30 & 0.30 & 0.30 & 0.30 & 0.30 \\
\hline L-Lysine $78 \%$ & 0.14 & 0.14 & 0.14 & 0.14 & 0.14 \\
\hline Vitamin premix & 0.10 & 0.10 & 0.10 & 0.10 & 0.10 \\
\hline Mineral premix & 0.10 & 0.10 & 0.10 & 0.10 & 0.10 \\
\hline L- Threonine $98.5 \%$ & 0.03 & 0.03 & 0.03 & 0.03 & 0.03 \\
\hline Choline Chloride $60 \%$ & 0.02 & 0.02 & 0.02 & 0.02 & 0.02 \\
\hline Coccidiostat & 0.06 & 0.06 & 0.06 & 0.06 & 0.06 \\
\hline *AZOMITE ${ }^{\circledR}$ & ---- & 0.25 & 0.50 & 0.75 & 1.00 \\
\hline \multicolumn{6}{|c|}{ Analysed Value of the nutrients } \\
\hline Moisture (\%) & 9.26 & 10.13 & 9.50 & 10.73 & 10.24 \\
\hline Crude Protein (\%) & 19.84 & 20.19 & 19.93 & 20.33 & 20.32 \\
\hline Crude Fibre (\%) & 5.00 & 4.81 & 5.07 & 4.77 & 4.60 \\
\hline Crude Fat (\%) & 8.44 & 9.27 & 9.75 & 9.95 & 8.61 \\
\hline Ash (\%) & 7.00 & 7.40 & 7.56 & 7.12 & 8.70 \\
\hline Salt (\%) & 0.32 & 0.29 & 0.31 & 0.32 & 0.33 \\
\hline
\end{tabular}


Survivability and European efficiency factor (EEF)

The survivability percentage of dietary treatment groups was not differing statistically. The broilers fed control diet had lowest value of EEF while $0.25,0.50,0.75$ and $1.00 \%$ AZOMITE ${ }^{\circledR}$ supplemented diets had higher value $(p<0.01)$ at $22-32$ days (Table 4$)$, however, it was not significant up to 3 weeks of age. Cumulative EEF of experimental broilers from 0 32 days of age revealed that AZOMITE $^{\circledR}$ supplemented diets increased EEF $(p<0.01)$ compared to control group. Increasing level of AZOMITE $^{\circledR}(0.25-1.00 \%)$ did not affect EEF.

\section{Gain cost}

The broilers in control diet had higher feed cost per unit of meat production (Table 4) while $0.25, \quad 0.50, \quad 0.75$ and $1.00 \% \quad$ AZOMITE $^{\circledR}$ supplemented diets had lower at 22-32 days $(p<0.01)$. Cumulative feed cost per unit of meat production of the experimental broilers from 0 32 days of age revealed that AZOMITE $^{\circledR}$ supplemented diets significantly lowered cost $(p<0.01)$ compared to control group. There was no significant difference observed among the treatment groups with increasing level of AZOMITE $^{\circledR}(0.25-1.00 \%)$.

\section{Edible meat yield characteristics}

The analyzed data in the Table $\mathbf{5}$ indicates that the treatments had no significant difference among dietary groups in respect to dressing percent, thigh, drumsticks and breast percentage. The obtained data showed that wing, head, neck, liver, gizzard and heart percentage were affected by dietary groups. The wing weight $(p<0.05)$ was significantly lower in 0.25 level of AZOMITE $^{\circledR}$, neck weight $(p<0.01)$ significantly decreased with the increases of dietary AZOMITE ${ }^{\circledR}$ and highest liver weight was observed at $0.25 \%$ level of AZOMITE $^{\circledR}(p<0.01)$. Gizzard weight was higher in absence of AZOMITE $^{\circledR}(p<0.05)$. The heart weight $(p<0.05)$ was significantly higher at $1.0 \%$ level of AZOMITE $^{\circledR}$. Contradictory result was showed by Hooge (2008) who reported significant improvement on dressing percentage $(p<0.05)$ and breast yield $(p<0.01)$ compared to negative control when AZOMITE ${ }^{\circledR}$ was fed to broiler.

Table 3: Growth performance of broilers supplemented with different level of AZOMITE ${ }^{\circledR}$

\begin{tabular}{|c|c|c|c|c|c|c|c|}
\hline \multirow{2}{*}{$\begin{array}{l}\text { Para } \\
\text { met } \\
\text { er }\end{array}$} & \multirow{2}{*}{$\begin{array}{l}\text { Age } \\
\text { (day) }\end{array}$} & \multicolumn{5}{|c|}{ Treatment ( $\%$ AZOMITE ${ }^{\circledR}$ ) } & \multirow{2}{*}{$\begin{array}{l}\text { Level } \\
\text { of } \\
\text { Sig. }\end{array}$} \\
\hline & & 0.00 & 0.25 & 0.50 & 0.75 & 1.00 & \\
\hline \multirow{3}{*}{$\begin{array}{l}\text { LBW } \\
\text { (g) }\end{array}$} & 0 & $46.66 \pm 0.09$ & $46.59 \pm 0.08$ & $46.7 \pm 0.07$ & $46.5 \pm 0.1$ & $46.5 \pm 0.1$ & NS \\
\hline & 21 & $823 \pm 9.4$ & $818 \pm 8.8$ & $842.1 \pm 8.61$ & $839 \pm 6.9$ & $828 \pm 10.8$ & NS \\
\hline & 32 & $1206^{c} \pm 22.7$ & $1545^{\mathrm{b}} \pm 25.9$ & $1607.52^{\mathrm{ab}} \pm 24.32$ & $1628^{\mathrm{a}} \pm 19.8$ & $1582^{\mathrm{ab}} \pm 8.6$ & $* *$ \\
\hline \multirow{3}{*}{$\begin{array}{l}\text { BWG } \\
\text { (g) }\end{array}$} & $0-21$ & $763 \pm 8.4$ & $765 \pm 11.1$ & $783.05 \pm 11.02$ & $792 \pm 6.9$ & $776 \pm 13.1$ & NS \\
\hline & $22-32$ & $378^{\mathrm{b}} \pm 23.5$ & $722^{\mathrm{a}} \pm 22.3$ & $765.43^{\mathrm{a}} \pm 20.92$ & $776^{\mathrm{a}} \pm 15.8$ & $748^{\mathrm{a}} \pm 9.8$ & $* *$ \\
\hline & $0-32$ & $1134^{c} \pm 25$ & $1481^{b} \pm 32.2$ & $1532.85^{\mathrm{ab}} \pm 21.31$ & $1569^{a} \pm 15.3$ & $1518^{\mathrm{ab}} \pm 19.7$ & ** \\
\hline \multirow{3}{*}{$\begin{array}{l}\text { ADG } \\
(\mathrm{g})\end{array}$} & $0-21$ & $37 \pm 0.45$ & $36.7 \pm 0.4$ & $37.88 \pm 0.41$ & $37 \pm 0.3$ & $37.2 \pm 0.5$ & NS \\
\hline & $22-32$ & $34.8^{\mathrm{c}} \pm 2.13$ & $66.1^{\mathrm{b}} \pm 1.8$ & $69.58^{\mathrm{ab}} \pm 1.90$ & $71^{\mathrm{a}} \pm 1.6$ & $68.6^{\mathrm{ab}} \pm 0.9$ & $* *$ \\
\hline & $0-32$ & $36.25^{c} \pm .71$ & $46.8^{b} \pm 0.8$ & $48.78^{\mathrm{ab}} \pm 0.76$ & $49^{a} \pm 0.6$ & $48^{\mathrm{ab}} \pm 0.3$ & $* *$ \\
\hline
\end{tabular}

$\mathrm{LBW}=$ Average Live Weight, BWG= Live Weight Gain, ADG= Average daily gain, $\mathrm{g}=$ Grams, NS= Nonsignificant, Values indicate average \pm Standard Error Mean (SEM). ${ }^{\mathbf{a}, \mathbf{b}, \mathbf{c}, \mathbf{d}}$ Means bearing uncommon superscripts in a row differ significantly $(\mathrm{p}<0.05) . * *=\mathrm{P}<0.01, *=\mathrm{P}<0.05$. 
Natural minerals on broiler performance

Table 4: Feed intake, FCR, EEF and gain cost of the broiler supplemented with different level of AZOMITE $^{\circledR}$

\begin{tabular}{|c|c|c|c|c|c|c|c|}
\hline \multirow{2}{*}{$\begin{array}{l}\text { Parame } \\
\text { ter }\end{array}$} & \multirow{2}{*}{$\begin{array}{l}\text { Age } \\
\text { (day) }\end{array}$} & \multicolumn{5}{|c|}{ Treatment (\% AZOMITE $\left.{ }^{\circledR}\right)$} & \multirow{2}{*}{$\begin{array}{l}\text { Level } \\
\text { of } \\
\text { Sig. }\end{array}$} \\
\hline & & 0.0 & 0.25 & 0.50 & 0.75 & 1.00 & \\
\hline & $0-21$ & $1280 \pm 13.9$ & $1263 \pm 8.4$ & $1277 \pm 5.6$ & $1289 \pm 5.5$ & $1277 \pm 9.04$ & NS \\
\hline \multirow{3}{*}{$\begin{array}{l}\text { FI } \\
\text { (g) }\end{array}$} & $22-32$ & $1406^{b} \pm 35.4$ & $1588^{\mathrm{a}} \pm 28.03$ & $1649^{\mathrm{a}} \pm 13.7$ & $1648.09^{\mathrm{a}} \pm 14.78$ & $1644^{\mathrm{a}} \pm 15.2$ & $* *$ \\
\hline & $0-32$ & $2690^{\mathrm{b}} \pm 44.8$ & $2855^{\mathrm{a}} \pm 31.8$ & $2927^{\mathrm{a}} \pm 12.47$ & $2940^{a} \pm 17.9$ & $2922^{a} \pm 19.7$ & $* *$ \\
\hline & $0-21$ & $1.68 \pm 0.02$ & $1.65 \pm 0.02$ & $1.63 \pm 0.02$ & $1.63 \pm 0.01$ & $1.65 \pm 0.02$ & NS \\
\hline \multirow[t]{3}{*}{$\begin{array}{l}\text { FCR } \\
\text { Actual }\end{array}$} & $22-32$ & $3.78^{\mathrm{a}} \pm 0.2$ & $2.2^{\mathrm{b}} \pm 0.04$ & $2.16^{\mathrm{b}} \pm 0.05$ & $2.13^{b} \pm 0.03$ & $2.2^{b} \pm 0.02$ & ** \\
\hline & $0-32$ & $2.38^{\mathrm{a}} \pm 0.06$ & $1.93^{b} \pm 0.03$ & $1.91^{\mathrm{b}} \pm 0.02$ & $1.88^{\mathrm{b}} \pm 0.01$ & $1.93^{b} \pm 0.02$ & $* *$ \\
\hline & $0-21$ & $1.57 \pm 0.01$ & $1.55 \pm 0.01$ & $1.53 \pm 0.02$ & $1.54 \pm 0.01$ & $1.56 \pm 0.02$ & NS \\
\hline \multirow[t]{2}{*}{$\begin{array}{l}\text { FCR } \\
\text { Adjusted }\end{array}$} & $22-32$ & $3.87^{\mathrm{a}} \pm 0.2$ & $2.22^{\mathrm{b}} \pm 0.05$ & $2.16^{\mathrm{b}} \pm 0.05$ & $2.14^{b} \pm 0.03$ & $2.2^{b} \pm 0.03$ & $* *$ \\
\hline & $0-32$ & $2.26^{\mathrm{a}} \pm 0.04$ & $1.86^{\mathrm{b}} \pm 0.02$ & $1.83^{b} \pm 0.02$ & $1.82^{b} \pm 0.01$ & $1.87^{b} \pm 0.02$ & $* *$ \\
\hline \multirow{2}{*}{$\begin{array}{l}\text { Survivab } \\
\text { ility }\end{array}$} & $0-21$ & $98 \pm 0.9$ & $99.02 \pm 0.62$ & $98 \pm 0.98$ & $100 \pm 0$ & $99 \pm 0.98$ & NS \\
\hline & $22-32$ & $99 \pm 0.6$ & $99.51 \pm 0.49$ & $100 \pm 0$ & $99 \pm 0.62$ & $99.5 \pm 0.52$ & NS \\
\hline \multirow{2}{*}{ (\%) } & $0-32$ & $97 \pm 1.3$ & $98.53 \pm 0.66$ & $98 \pm 0.98$ & $99 \pm 0.62$ & $98.5 \pm 1.47$ & NS \\
\hline & $0-21$ & $229 \pm 5.2$ & $233 \pm 5.7$ & $241 \pm 6.2$ & $245 \pm 3.63$ & $237.47 \pm 6.91$ & NS \\
\hline \multirow[t]{3}{*}{ EEF } & $22-32$ & $92.6^{b} \pm 9.9$ & $298^{\mathrm{a}} \pm 15.4$ & $323^{a} \pm 16.5$ & $329^{\mathrm{a}} \pm 11.54$ & $308.65^{a} \pm 7.42$ & $* *$ \\
\hline & $0-32$ & $154.7^{b} \pm 6.6$ & $247^{\mathrm{a}} \pm 9.2$ & $258^{\mathrm{a}} \pm 6.91$ & $268^{\mathrm{a}} \pm 4.03$ & $253^{a} \pm 6.72$ & $* *$ \\
\hline & $0-21$ & $76.9 \pm 0.6$ & $76.5 \pm 0.58$ & $75.39 \pm 0.7$ & $76.19 \pm 0.5$ & $77.2 \pm 0.7$ & NS \\
\hline \multirow{2}{*}{$\begin{array}{l}\text { Gain } \\
\text { cost } \\
\text { (BDT) }\end{array}$} & $22-32$ & $170.8^{\mathrm{a}} \pm 8.99$ & $99.9^{b} \pm 1.99$ & $98.3^{b} \pm 2.2$ & $96.95^{b} \pm 1.4$ & $100.5^{b} \pm 1.01$ & $* *$ \\
\hline & $0-32$ & $147.9^{\mathrm{a}} \pm 4.30$ & $93.3^{\mathrm{b}} \pm 1.41$ & $91.94^{b} \pm 1.5$ & $91.04^{b} \pm 1.3$ & $93.2^{b} \pm 0.5$ & $* *$ \\
\hline
\end{tabular}

$\mathrm{EEF}=$ European efficiency factor, NS= Non-significant, Values indicate average \pm Standard Error Mean (SEM). $\mathbf{a}, \mathbf{b}, \mathbf{c}, \mathbf{d}$ Means bearing uncommon superscripts in a row differ significantly $(\mathrm{p}<0.05)$. $* *=\mathrm{P}<0.01, *=\mathrm{P}<0.05$.

\section{Conclusion}

The finding of present study suggests that AZOMITE $^{\circledR}$ enacted positive effects on growth performance of commercial broiler at $0.25,0.5$, 0.75 and $1.0 \%$. Feeding AZOMITE $^{\circledR}$ at $0.5,0.75$ and $1.0 \%$ consistently improved LBW, BWG and ADG, compared to $0.0 \%$ and $0.25 \%$. This observation could aid in the affordability of AZOMITE ${ }^{\circledR}$ for producers, increasing its feasibility as a partial supplementation of minerals at 0.5 to $1.0 \%$ for commercial broiler production.

\section{Acknowledgements}

We would like to acknowledge C. P. Bangladesh Co., Ltd for providing logistics supports to conduct the experiment and department of Poultry Science for providing all sorts of technical supports.

\section{Conflict of Interest}

There is no conflict of interest to declare. 
Zubayer et al., (2019) Bang. J. Anim. Sci. 48 (2):92-98

Table 5: Meat yield characteristics of broilers fed diets supplemented with AZOMITE ${ }^{\circledR}$

\begin{tabular}{|c|c|c|c|c|c|c|}
\hline \multirow[b]{2}{*}{ Parameter } & \multicolumn{5}{|c|}{ Treatment (level of AZOMITE ${ }^{\circledR}$ in \%) } & \multirow{2}{*}{$\begin{array}{l}\text { Lev } \\
\text { el } \\
\text { of } \\
\text { Sig }\end{array}$} \\
\hline & 0.00 & 0.25 & 0.50 & 0.75 & 1.00 & \\
\hline $\begin{array}{l}\text { Live body } \\
\text { wt }(\mathrm{g} / \mathrm{b})\end{array}$ & $1404.67^{\mathrm{C}} \pm 30.24$ & $1547.42^{b} \pm 11.95$ & $1627.83^{\mathrm{a}} \pm 1.61$ & $1636.58^{\mathrm{a}} \pm 11.67$ & $1596.08^{\mathrm{a}} \pm 4.70$ & ** \\
\hline $\begin{array}{l}\text { Dressing wt } \\
(\mathrm{g} / \mathrm{b})\end{array}$ & $948.2^{d} \pm 17.85$ & $1060.65^{\mathrm{c}} \pm 9.21$ & $1119.09^{\mathrm{ab}} \pm 3.19$ & $1128.79^{a} \pm 8.99$ & $1084.92^{b c} \pm 9.12$ & $* *$ \\
\hline $\begin{array}{l}\text { Dressing wt } \\
(\%)\end{array}$ & $67.73 \pm 1.53$ & $68.56 \pm 0.48$ & $68.75 \pm 0.63$ & $68.98 \pm 0.42$ & $67.98 \pm 0.58$ & NS \\
\hline $\begin{array}{l}\text { Thigh wt } \\
(\%)\end{array}$ & $10.25 \pm 0.31$ & $10.65 \pm 0.12$ & $10.42 \pm 0.16$ & $10.58 \pm 0.11$ & $10.49 \pm 0.19$ & NS \\
\hline $\begin{array}{l}\text { Drumstick } \\
\text { wt. (\%) }\end{array}$ & $8.7 \pm 0.20$ & $8.43 \pm 0.07$ & $9.01 \pm 0.13$ & $8.92 \pm 0.12$ & $8.68 \pm 0.18$ & NS \\
\hline $\begin{array}{l}\text { Breast wt. } \\
(\%)\end{array}$ & $19.35 \pm 0.62$ & $18.79 \pm 0.41$ & $19.41 \pm 0.46$ & $18.38 \pm 0.44$ & $18.59 \pm 0.46$ & NS \\
\hline $\begin{array}{l}\text { Wing wt. } \\
(\%)\end{array}$ & $7.23^{\mathrm{ab}} \pm 0.12$ & $7.03^{b} \pm 0.13$ & $7.47^{a} \pm 0.12$ & $7.51^{a} \pm 0.14$ & $7.47^{a} \pm 0.12$ & $*$ \\
\hline $\begin{array}{l}\text { Head wt. } \\
(\%)\end{array}$ & $2.4^{b} \pm 0.04$ & $2.31^{b} \pm 0.04$ & $2.33^{b} \pm 0.03$ & $2.37^{b} \pm 0.05$ & $2.57^{\mathrm{a}} \pm 0.06$ & ** \\
\hline $\begin{array}{l}\text { Neck wt. } \\
(\%)\end{array}$ & $2.36^{\mathrm{a}} \pm 0.12$ & $2.0^{b} \pm 0.06$ & $1.97^{b} \pm 0.08$ & $1.95^{\mathrm{b}} \pm 0.04$ & $1.85^{\mathrm{b}} \pm 0.05$ & ** \\
\hline $\begin{array}{l}\text { Liver wt. } \\
(\%)\end{array}$ & $2.32^{\mathrm{ab}} \pm 0.10$ & $2.37^{\mathrm{a}} \pm 0.12$ & $2.02^{c} \pm 0.06$ & $2.08^{b c} \pm 0.05$ & $2.1^{b c} \pm 0.06$ & $*$ \\
\hline $\begin{array}{l}\text { Gizzard wt. } \\
(\%)\end{array}$ & $2.17^{a} \pm 0.08$ & $1.96^{b} \pm 0.05$ & $1.94^{b} \pm 0.08$ & $1.83^{b} \pm 0.06$ & $1.86^{\mathrm{b}} \pm 0.09$ & $*$ \\
\hline $\begin{array}{l}\text { Heart wt. } \\
(\%)\end{array}$ & $0.42^{b} \pm 0.02$ & $0.4^{b} \pm 0.01$ & $0.4^{b} \pm 0.01$ & $0.44^{a b} \pm 0.01$ & $0.47^{a} \pm 0.02$ & $*$ \\
\hline
\end{tabular}

NS $=$ Non-significant, Values indicate average \pm Standard Error Mean (SEM). ${ }^{a, b, c}$ Means bearing dissimilar superscript in a row differ significantly $(\mathrm{p}<0.05), * *=\mathrm{P}<0.01, *=\mathrm{P}<0.05$.

\section{References}

Hays VW and MJ Swenson (1985). Minerals and Bones. Dukes' Physiology of Domestic Animals 449-466.

Hooge D (2008). Natural Minerals Can Benefit Broiler Diets. Feedstuffs 80:24-26.

Karovic D, V Djermanovic, S Mitrovic, V Radovic, D Okanovic and V Djekic (2013). The effect of mineral adsorbents in poultry production. World's Poultry Science Journal 69:335-342.

Khatun A, SD Chowdhury, BC Roy, B Dey, A Haque and B Chandran (2018). Comparative effects of inorganic and three forms of organic trace minerals on growth, carcass traits, immunity, and profitability of broilers. Journal of Advance Veterinary and Animal Research 6(1):66-73.

Liu HK, BD Green, NH McClenaghan, JT McCluskey and PR Flatt (2004). Long-term beneficial effects of vanadate, tungstate and molybdate on insulin secretion and function of cultured beta cells. Pancreas, 28:364-368. Malhotra VK (1998). Biochemistry for Students. $10^{\text {th }}$ Edition Jaypee Brothers Medical Publishers (P) Ltd, India.

McDowell LR (2003). Minerals in Animal and Human Nutrition.2nd Edition, Elsevier Science, BV Amsterdam, Netherlands.

McNaughton JL, D Fodge, WW Emerson and DM Hooge (2011). Live performance and energy uplift of $0.5 \%$ Azomite Feed-Grit (versus 0\%) were evaluated in broiler feeds in two series with increasing level of metabolizable energy under disease stresses in floor pens. Poultry Science 90 (E-Suppl. 1)

Nielsen FH (1985). The importance of diet composition in ultra-trace element research. Journal of Nutrition 11:1239-1247.

Nielsen FH (1996). How should dietary guidance be given for mineral elements with beneficial actions or suspected of being essential? Journal of Nutrition 126:2377-2385.

Ozcan M (2003). Mineral Contents of some Plants used as condiments in Turkey. Food Chemistry 84:437-440.

Qinghua V (1996). Winning world-wide. Feeding Times 1:6-7.

Steel RGD and JH Torrie (1980). Principles and Procedures of Statistics: A Biomedical Approach. $2^{\text {nd }}$ Edition, McGraw-Hill Book Co., Toronto, Canada.

Underwood EJ (1971). Trace Elements in Human and Animal Nutrition, 3rd Edition, Academic Press, New York, USA.pp. 116.

Uthus EO and CD Seaborn (1996). Deliberations and evaluations of the approaches, endpoints and paradigms for dietary recommendations of the other trace elements. Journal of Nutrition 126:2452-2459. 